\title{
Inhibitory effect of coated mannan against the adhesion of Candida biofilms to denture base resin
}

\author{
Maki SATO ${ }^{1}$, Tomoko OHSHIMA², Nobuko MAEDA² and Chikahiro OHKUBO ${ }^{1}$ \\ ${ }^{1}$ Department of Removable Prosthodontics, Tsurumi University School of Dental Medicine, 2-1-3 Tsurumi, Tsurumi-ku, Yokohama, Kanagawa 230- \\ 8501, Japan \\ ${ }^{2}$ Department of Oral Microbiology, Tsurumi University School of Dental Medicine, 2-1-3 Tsurumi, Tsurumi-ku, Yokohama, Kanagawa 230-8501, \\ Japan \\ Corresponding author, Maki SATO; E-mail: sato-maki@tsurumi-u.ac.jp
}

\begin{abstract}
The adherence of Candida on dentures is related to diseases such as denture stomatitis and aspiration pneumonia. Mannan is a major component of the Candida cell surface, and contributes to the cell adherence. A previous report indicated that the adherence of C. albicans to culture dishes was inhibited by the coating them with mannan. The purpose of this study was to examine the adhesion inhibitory effect of mannan coating on acrylic denture surfaces against C. albicans and C. glabrata. The amount of Candida attached on the acrylic surfaces coated with mannan was calibrated by culture methods. Mannan showed significant inhibitory effects on Candida adhesion in both the yeast and hyphal form in a concentration-dependent manner, and the durability of the inhibitory effect continued for three days. These results suggest that mannan coating on the denture base acrylic can prevent Candida adhesion on the denture.
\end{abstract}

Keywords: Candida albicans, Candida glabrata, Mannan, Denture base resin

\section{INTRODUCTION}

The surface of denture base acrylic resin is porous, and denture plaque easily adheres to the surface of dentures. The colonization of microorganisms on the denture base acrylic resin occurs rapidly, and Candida species adhere strongly to denture base materials ${ }^{11}$.

The prevalence of denture wearing was $49 \%$ in subjects aged 15 years and over, and $96.9 \%$ of these subjects were elderly, according to a survey of dental diseases in $\mathrm{Japan}^{2}$. It has been reported that the carriage rate of Candida species in the oral cavity is increased by denture wearing, and Candida albicans and Candida glabrata were often detected ${ }^{3-5}$. The adhesion of Candida species to denture surfaces is an important pathogenic factor not only because it induces the degradation of the denture base material, but also because it can cause denture stomatitis. Furthermore, poor denture hygiene might increase the risk of aspiration pneumonia in the elderly ${ }^{6}$.

Denture stomatitis is a very common disorder affecting denture wearers, and it has been reported in 11$67 \%$ of complete denture wearers ${ }^{7}$. Antifungal therapy is effective for the acute treatment of inflammation associated with denture stomatitis. However, the effectiveness of antifungal treatment is limited longterm, and rapid recurrence of denture stomatitis can occur shortly after stopping treatment. The recurrence of this disorder seems to be due to continuous infection by residual Candida species on the denture surfaces ${ }^{6,8}$. The surface hydrophobicity of the materials increases the ability of the hyphal forms of $C$. albicans to colonize the denture surfaces, and in vitro studies have shown that the hydrophilic coating materials can decrease the adhesion of C. albicans, but not other Candida species, thereby decreasing their ability to colonize the denture material surface ${ }^{6,9)}$.

The outermost layer of the Candida cell wall is covered with hydrophilic polysaccharides, such as mannan or galactomannan ${ }^{10)}$. These mannans on the fungal surface function as adhesins, which are involved not only in the adhesion to the host cell ${ }^{11,12}$ but also in the adsorption to plastic plates. On the other hand, when the plastic surfaces of culture dishes were coated with mannan, the adherence of $C$. albicans to the dishes was significantly inhibited ${ }^{13,14)}$.

The $\beta$-1, 2-linked mannooligosaccharide in mannan shows strong antigenicity and is associated with the induction of tumor necrosis factor alpha (TNF-a), a proinflammatory cytokine ${ }^{10,15}$. Mannan also promotes wound healing by increasing the proliferation of fibroblasts and vascular endothelial cells, and by enhancing the production of collagen ${ }^{16}$. Recently, food and medical devices coated with mannan have been developed, and a variety of effects of mannan have been confirmed ${ }^{17-19)}$. Based on the findings of these clinical reports, mannan appears to be effective for preventing the development of local and systemic diseases, including denture stomatitis.

The purpose of this study was to examine whether adding a mannan coating on the denture base acrylic surfaces can inhibit the adhesion of C. albicans and C. glabrata, which are often identified from patients with denture stomatitis, and to evaluate the duration of this effect of mannan on the adhesion of $C$. albicans. 


\section{MATERIALS AND METHODS}

Preparation of denture base acrylic resin specimens Acryl fluid resin for clear type dentures (Palapress Vario, Kulzer, Germany) was polymerized according to the manufacturer's instructions, and was trimmed into $3 \times 3 \times 2 \mathrm{~mm}$ pieces for the REDOX indicator assay and $25 \times 25 \times 2 \mathrm{~mm}$ pieces for the adherence inhibitory assay. All samples were sterilized with $80 \%$ ethanol, and immersed in sterilized distilled water (DW).

\section{Preparation of reagents and coatings}

Purified mannan derived from Saccharomyces cerevisiae (Sigma Chemical Co., MO, USA) at concentrations of 0.1 $\mathrm{mg} / \mathrm{mL}, 1 \mathrm{mg} / \mathrm{mL}, 10 \mathrm{mg} / \mathrm{mL}$, and $10 \mathrm{mg} / \mathrm{mL}$ of mannose (Wako Pure Chemical, Osaka, Japan) were used in this study. All of the solutions were sterilized by filtration. Sterilized acrylic pieces were immersed in the above three concentrations of mannan solution (mannan group) or mannose solution (mannose group) overnight. The control group (DW) was immersed in DW overnight. The saliva group was immersed in DW overnight and put into filtered human saliva. After immersion, all of the samples except for the control, were placed into the filtered human saliva, and the acrylic pieces were kept in saliva overnight.

\section{Candida strains and culture conditions}

1. Candida albicans

Candida albicans ATCC18804 was cultivated in Tryptic Soy Broth supplemented with 5\% dextrose (TSBD; Becton, Dickinson and Company, NJ, USA) medium at $37^{\circ} \mathrm{C}$ with shaking for preculture. Two different media were used for $C$. albicans cultivation. For the hyphal form of $C$. albicans, a $300 \mu \mathrm{L}$ aliquot of the preculture was inoculated into $3 \mathrm{~mL}$ of RPMI1640 medium supplemented with $10 \%$ fetal bovine serum (FBS; GIBCO, Invitrogen Co., CA, USA). For the yeast form of $C$. albicans, a $300 \mu \mathrm{L}$ aliquot of the preculture was inoculated into $3 \mathrm{~mL}$ of TSBD. Both were cultivated with the acrylic pieces for $72 \mathrm{~h}$. The number of $C$. albicans cells was adjusted to approximately $1 \times 10^{6}$ to $1 \times 10^{7} \mathrm{CFU} / \mathrm{mL}$ in the precultures.

\section{Candida glabrata}

Candida glabrata ATCC90030 was cultivated in TSBD medium at $37^{\circ} \mathrm{C}$ with shaking for precultures. The number of $C$. glabrata cells was adjusted to approximately $1 \times 10^{7}$ to $1 \times 10^{8} \mathrm{CFU} / \mathrm{mL}$. A $300 \mu \mathrm{L}$ of aliquot of the preculture was inoculated into TSBD with the acrylic pieces for $72 \mathrm{~h}$.

Adherence inhibitory assay of the yeast form of Candida cells using the washing out method

The washing out method was slightly modified to use JIS Z 2801 as the antibiotic. After immersion into each mannan solution $(0.1 \mathrm{mg} / \mathrm{mL}, 1 \mathrm{mg} / \mathrm{mL}, 10 \mathrm{mg} / \mathrm{mL})$ or DW (control) overnight, $100 \mu \mathrm{L}$ aliquots of $C$. albicans or $C$. glabrata suspensions, adjusted to approximately $1 \times 10^{6}$ to $1 \times 10^{7} \mathrm{CFU} / \mathrm{mL}$ with TSBD, were dropped onto acrylic pieces, and the plates were covered with sterilized polyethylene film $(20 \times 20 \mathrm{~mm})$. These acrylic pieces were incubated at $30^{\circ} \mathrm{C}$ for $72 \mathrm{~h}$. After incubation, the acrylic pieces were washed with distilled water to remove nonadherent Candida cells. The adherent Candida were washed out from each of the acrylic pieces with the Soya casein digest lecithin polysorbate (SCDLP; Wako Pure Chemical, Osaka, Japan) medium, and the cell count in the washout liquid from the SCDLP was measured by a CFU assay.

\section{REDOX indicator assay}

A $300 \mu \mathrm{L}$ aliquot of C. albicans or C. glabrata suspension culture was added into each $15 \mathrm{~mL}$ tube containing five acrylic pieces, and $3 \mathrm{~mL}$ of RPMI 1640 medium was used for $C$. albicans, while TSBD medium was used for $C$. glabrata. These tubes were placed in a shaker incubator for three to seven days at $37^{\circ} \mathrm{C}$. After incubation, the acrylic pieces were immersed and washed in distilled water three times to remove non-adherent cells. The washed pieces were placed on 96 well plates, and 150 $\mu \mathrm{L}$ of the RPMI 1640 medium supplemented with 10\% FBS and a REDOX indicator (Alamar blue; Trek Diagnostic Systems, Cleveland, OH, USA) was added, and were incubated again. The $C$. glabrata group was supplemented with $50 \mu \mathrm{L}$ of $20 \%$ glucose, in addition to the RPMI 1640 medium containing the REDOX indicator.

The amount of adherent Candida was estimated based on the fluorescence levels (EX: 530, EM: 590) by a Fluorescent Microplate Reader Cyto-Fluor 2 (Per Septive Biosystems, Farmingham, MA, USA). The durability of the mannan effect was examined every day for one week.

To confirm the adherence of $C$. albicans biofilms, the acrylic pieces from the control group and $10 \mathrm{mg} / \mathrm{mL}$ mannan group were observed with microscopy at $400 \times$ magnification after Gram staining.

\section{Statistical analysis}

The statistical analyses were performed among multiple groups using the Kruskal-Wallis test, and between two groups using a Mann-Whitney U-test (SPSS $14.0 \mathrm{~J}$ ). Significant differences were considered to be indicated by values of $p<0.05$.

\section{RESULTS}

Adherence inhibitory assay of the yeast form of C. albicans cells by the washing out method

The amounts of the adherent yeast form of C. albicans detected by the washing out method are shown in Fig. 1. The CFU of $C$. albicans yeast cells were significantly decreased in a mannan concentration-dependent manner $(p<0.05)$. There were significant differences between each of the mannan groups and the control, and among the mannan groups $(p<0.05)$. 


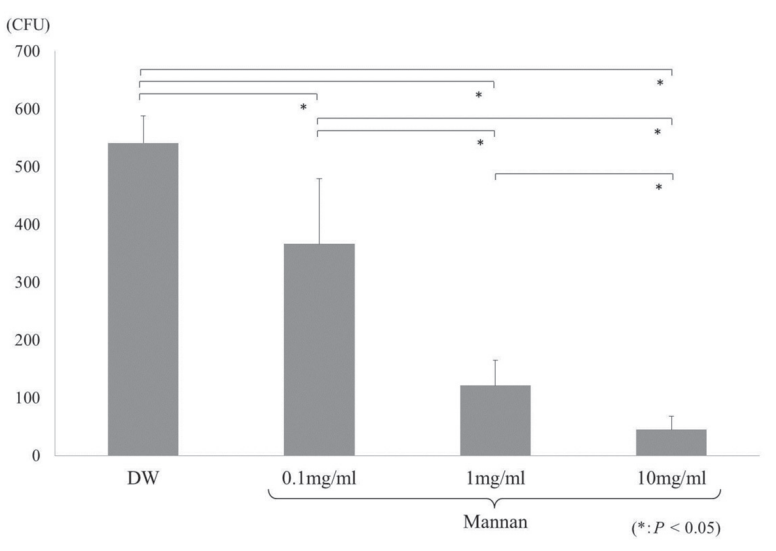

Fig. 1 The amount of adhered yeast form of C. albicans detected by washing out method.

There was significantly difference between two groups (Mann-Whitney U-test, $p<0.05$ ).

Inhibition of the adherence of the hyphal form of C. albicans as indicated by the REDOX indicator assay The amount of $C$. albicans that adhered to the acrylic surfaces after incubation for $30 \mathrm{~h}$ is shown in Fig. 2a. In the control group, the saliva group and the mannose group, a large amount of $C$. albicans biofilm was noted to be present on all acrylic pieces, but there was no significant difference among these groups. The fluorescence level of the $C$. albicans that adhered to the acrylic pieces treated with $0.1 \mathrm{mg} / \mathrm{mL}$ of mannan was $45 \%$ of the level in the control group, and was $12 \%$ on the pieces treated with 1 $\mathrm{mg} / \mathrm{mL}$ mannan, and only $1 \%$ on the pieces treated with $10 \mathrm{mg} / \mathrm{mL}$ mannan. There were significant differences between each of the mannan groups and the control, the saliva group and the mannose group, except for between the saliva group and the $0.1 \mathrm{mg} / \mathrm{mL}$ of mannan group $(p<0.05)$.

A micrograph of the C. albicans biofilms after Gram staining is shown in Fig. 2b. In the control group, a larger amount of $C$. albicans biofilms was observed on the acrylic pieces compared to those treated with $10 \mathrm{mg} /$ $\mathrm{mL}$ of mannan.

The durability of the effect of mannan on C. albicans as determined by the REDOX indicator assay

The durability of the effect of the mannan treatments is shown in Fig. 3. The level of fluorescence in the mannan groups was significantly decreased $(p<0.05)$ in a mannan concentration-dependent manner on the first and second days, but the level was not decreased compared to the control group on the third, fourth or fifth days. On the third day, the control, $0.1 \mathrm{mg} / \mathrm{mL}$ and $1 \mathrm{mg} / \mathrm{mL}$ of mannan samples were significantly different from the samples treated with $10 \mathrm{mg} / \mathrm{mL}$ of mannan $(p<0.05)$. On the fourth and fifth days, there were no significant differences among the test groups. In the control group, the amount of fluorescence was the same for one week. a

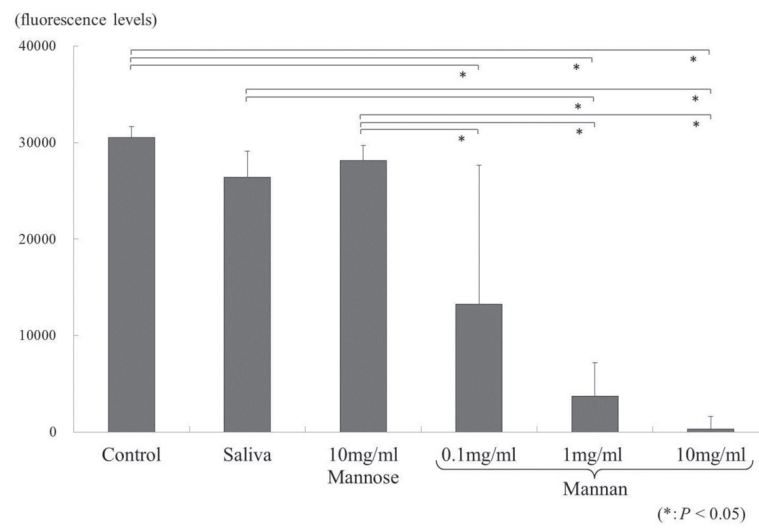

b

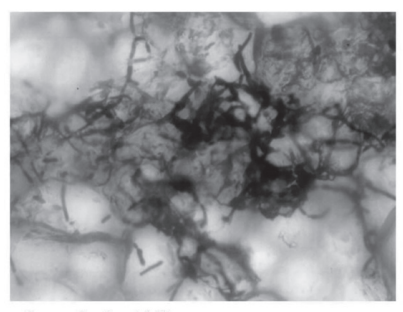

Control $(\times 400)$

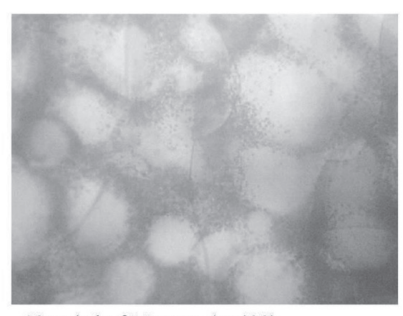

$10 \mathrm{mg} / \mathrm{ml}$ of Mannan $(\times 400)$

Fig. 2 The amount of adhered hyphal form of C. albicans on resin surfaces.

a: Redox indicator assay of hyphal form of $C$. albicans

Statistical analyzes of fluorescence levels were performed among multigroups by Kruskal-Wallis, and between two groups of control and each test group was analyzed by Mann-Whitney U-test, significant differences were judged as $p<0.05$.

b: Micrograph of $C$. albicans biofilms after gram staining

In control group, a large amount of C. albicans biofilms on the acryl pieces were observed compared with $10 \mathrm{mg} / \mathrm{mL}$ of the mannan group.

Adherence inhibitory assay of the yeast form of $C$. glabrata cells by the washing out method

The amounts of the adherent yeast form of C. glabrata detected by the washing out method are shown in Fig. 4a. In the control group and $0.1 \mathrm{mg} / \mathrm{mL}$ of mannan, a large amount of $C$. glabrata biofilms was observed, and the CFU level was almost the same level as that of $C$. albicans (Fig. 4a). There was a significant difference between the $10 \mathrm{mg} / \mathrm{mL}$ of mannan group and the control groups or the $0.1 \mathrm{mg} / \mathrm{mL}$ of mannan group $(p<0.05)$, but there were no significant differences among the mannan groups.

Inhibition of the adherence of C. glabrata by mannan, as determined by the REDOX indicator assay

In the control group, the saliva group and the mannose group, a large amount of C. glabrata biofilms was observed, and the fluorescence level was almost the same 


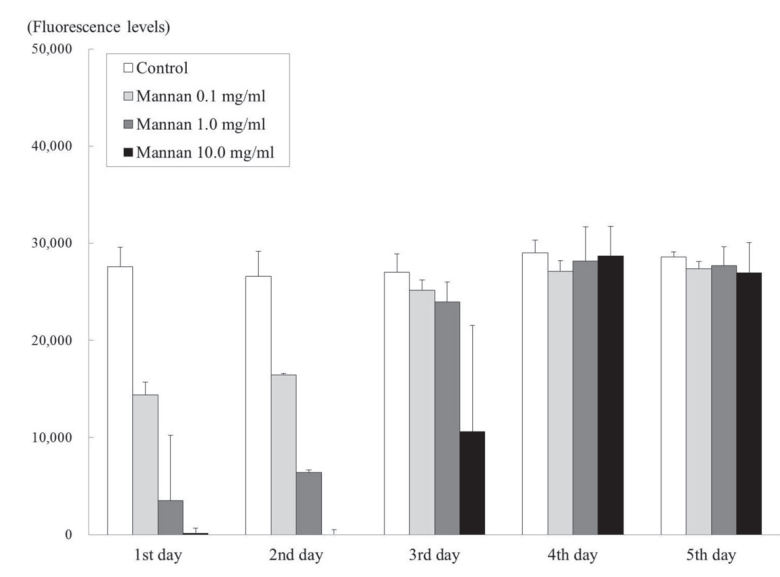

Fig. 3 The duration effect of Mannan by measuring the amount of hyphal form of $C$. albicans adhesion to acrylic surfaces coated with mannan.

Statistical analyzes were performed among multigroups in a day or multigroups in concentration by Kruskal-Wallis, and between each groups by Mann-Whitney U-test (Significant differences were judged as $p<0.05)$. In the first and second day, all test groups in amount of fluorescence were significant different except for between $0.1 \mathrm{mg} / \mathrm{mL}$ and $1 \mathrm{mg} / \mathrm{mL}$ of mannan in the second day. In the third day, control, $0.1 \mathrm{mg} /$ $\mathrm{mL}$ and $1 \mathrm{mg} / \mathrm{mL}$ of mannan were significantly different between $10 \mathrm{mg} / \mathrm{mL}$ of mannan $(p<0.05)$.

level as that of $C$. albicans (Fig. 4b). The fluorescence levels of $C$. glabrata that adhered to the pieces treated with $0.1 \mathrm{mg} / \mathrm{mL}$ of mannan was $62 \%$ the level of the control group, and was $40 \%$ for the pieces treated with $1 \mathrm{mg} / \mathrm{mL}$, and 5\% for samples treated with $10 \mathrm{mg} / \mathrm{mL}$. There was a significant difference between the $10 \mathrm{mg} /$ $\mathrm{mL}$ of mannan group and the control, the saliva and mannose groups $(p<0.05)$, but there were no significant differences among the mannan groups.

\section{DISCUSSION}

The outermost layer of the cell wall of Candida consists of a complex of hydrophilic polysaccharides, such as galactomannan and mannan, which are involved in the adhesion of the Candida to host cells, as well as the antigenicity $^{11,12)}$. Iwamoto et al. reported that mannan from $S$. cerevisiae had an inhibitory effect on the adhesion of $C$. albicans to plastic culture dish surfaces ${ }^{13,14)}$. They examined only the adhesion inhibitory effect for the hyphal form of $C$. albicans, not the yeast form. The biofilms of $C$. albicans consist of both the yeast and hyphal forms, therefore, inhibiting the adhesion of both forms is important ${ }^{3,20)}$.

Recently, C. glabrata was not only reported to have a high detection rate in the oral cavity of elderly subjects, but was also identified as a pathogen causing denture stomatitis. C. glabrata frequently acquires
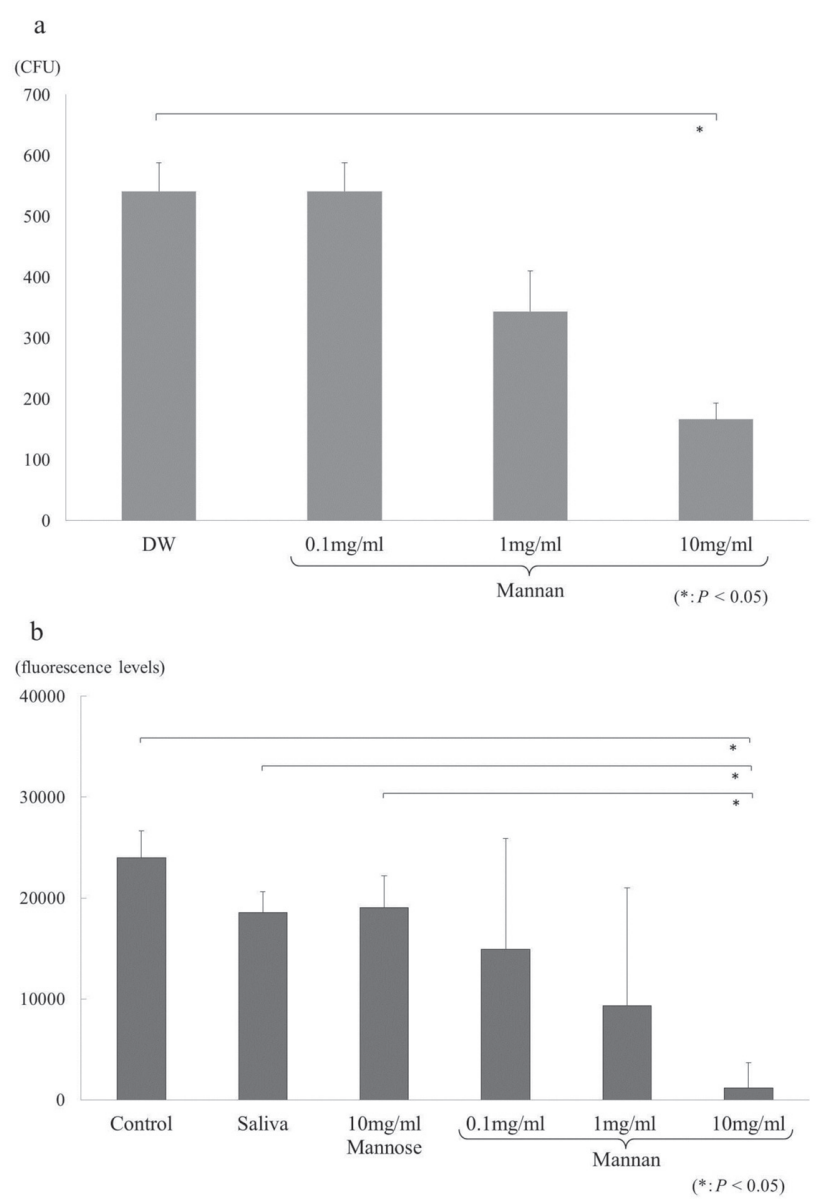

Fig. 4 The amount of adhered C. glabrata to mannan treated resin surfaces.

a: The detection by washing out method

There was significantly difference between two groups. (Mann-Whitney U-test, $p<0.05$ ).

b: The detection by Redox indicator assay

Statistical analyzes were performed among multigroups by Kruskal-Wallis, and between two groups of the control group and each test group was analyzed by Mann-Whitney U-test, significant differences were judged as $p<0.05$.

resistance to antifungal drugs, making it difficult to eradicate $^{21)}$. In this study, we examined the inhibitory effect of a mannan coating on the denture base acrylic surface against the adhesion of both of $C$. albicans and C. glabrata. In addition, since it is important to know how long the inhibitory effect of the mannan coating will be maintained for clinical applications, we carried out a durability test of the mannan against $C$. albicans adhesion.

The adhesion of the yeast form of $C$. albicans was inhibited in a mannan concentration-dependent manner, as indicated by experiments using a modified JIS Z 2801 method (Fig. 1). Since the yeast form of C. albicans often switches to the hyphal form in the oral cavity ${ }^{20)}$, we also 
wanted to examine the effects on the hyphal form of $C$. albicans. However, the hyphal form of $C$. albicans could not be recovered by the washing out method even in the control group, because C. albicans was too strongly attached on the surface of the denture base acrylic pieces to be released into the washing medium (data not shown). Therefore, the detection system was changed to a method monitoring the viable microbial count by examining the metabolism using the REDOX indicator. As the REDOX indicator in the medium measures the metabolism based on the amount of fluorescence produced, it reflects the number of surviving microorganisms ${ }^{22)}$.

From the results shown in Figs. $2 \mathrm{a}$ and $4 \mathrm{~b}$, it can be concluded that mannan inhibited the adhesion of Candida in a concentration-dependent manner, but mannose was not able to inhibit Candida adhesion even at a high concentration. Iwamoto et al. reported that the adhesion inhibitory effect was observed after coating the surface with $1 \mathrm{mg} / \mathrm{mL}$ of mannan for two hours, but that there was not an inhibitory effect for $0.1 \mathrm{mg} / \mathrm{mL}$ of mannan ${ }^{14)}$. In this study, the application of $0.1 \mathrm{mg} / \mathrm{mL}$ of mannan coating overnight showed an inhibitory effects on the adhesion of the hyphal form of C. albicans (Fig. $2 a)$. The difference between these results was likely due to the coating time.

In the case of $C$. glabrata, the inhibitory effect was also observed to occur in a concentration-dependent manner (Figs. $4 \mathrm{a}$ and $4 \mathrm{~b}$ ), and the $10 \mathrm{mg} / \mathrm{mL}$ of mannan led to significantly higher anti-adhesive effects. This indicates that mannan effectively prevents the adhesion of two major Candida species to the denture surface, indicating the possibility of applying such a coated for clinical dentistry.

Recently, mannan binding molecules on the surface dendritic cells, Dectin-2 and DC-sign, were found ${ }^{23-26)}$. In dendritic cells and macrophages, the expression of inflammatory cytokines such as TNF- $\alpha$ is induced by the binding of the mannooligosaccharide in mannan ${ }^{10,15)}$. However, dendritic cells are generally not exposed to the outermost layer of the oral mucosa. Therefore, no immune response should be induced in the oral cavity due to its separation by the epithelium, and thus, inflammation should not be induced by coating denture surfaces with mannan. Additionally, there were some contradictory reports about the role of mannan in inflammation. For example, one report indicated that mannan promotes wound healing by increasing the proliferation of fibroblasts and vascular endothelial cells, and by enhancing the production of collagen ${ }^{16)}$, and foods and medical devices including mannan have been developed based on these properties.

Although there is much demand for acrylic resin base dentures in aging societies, they are difficult to keep clean. Mannan has been applied widely in the medical field, particularly for the prevention of influenza and bacterial infections, inflammation and allergic diseases ${ }^{27-29}$. Based on the previous results and those from the present study, we advocate the use of mannan in the clinical practice of dentistry, as employing a mannan coating on dental devices might improve the oral hygiene. However, in this study, the inhibitory effect of the mannan coating on denture resin pieces against Candida adhesion lasted for three days. It is therefore necessary to investigate methods to stabilize mannan, such as the cross-linking between mannan molecules and the denture base acrylic pieces, in the future.

In conclusion, our results suggest that applying a mannan coating on the denture base acrylic resin can prevent the adhesion of C. albicans and C. glabrata.

\section{REFERENCES}

1) Nalbant AD, Kalkanci A, Filiz B, Kustimur S. Effectiveness of different cleaning agents against the colonization of Candida spp and the in vitro detection of the adherence of these yeast cells to denture acrylic surfaces. Yonsei Med J 2008; 49: 647654 .

2) STATISTICAL TABLES of THE SURVEY OF DENTAL DISEASES (2011) Part 1. Table IV-1-1 Prevalence of prostheses in permanent teeth, by type of prostheses, sex and age group (15 years of age and over), <http://www.mhlw. go.jp/toukei/list/dl/62-17c23-1.pdf >; 2011

3) Nikawa H, Mikihira S, Egusa H, Fukushima H, Kawabata R, Hamada T, Yatani H. Candida adherence and biofilm formation on oral surfaces. Jpn J Med Mycol 2005; 46: 233242.

4) Nikawa H, Hamada T, Yamamoto T. Denture plaque — past and recent concerns-. J Dent 1998; 26: 299-304.

5) Ribeiro DG, Pavarina AC, Dovigo LN, Machado AL, Giampaolo ET, Vergani CE. Prevalence of Candida spp. associated with bacteria species on complete dentures. Gerodontology 2012; 29: 203-208.

6) Gendreau L, Loewy ZG. Epidemiology and etiology of denture stomatitis. J Prosthodont 2011; 20: 251-260.

7) Arendorf TM, Walker DM. Denture stomatitis: a review. J Oral Rehabil 1987; 4: 17-27.

8) Chandra J, Mukherjee PK, Leidich SD, Faddoul FF, Hoyer LL, Douglas LJ, Ghannoum MA. Antifungal resistance of candidal biofilms formed on denture acrylic in vitro. J Dent Res 2001; 80: 903-908.

9) Yoshijima Y, Murakami K, Kayama S, Liu D, Hirota $\mathrm{K}$, Ichikawa T, Miyake Y. Effect of substrate surface hydrophobicity on the adherence of yeast and hyphal Candida. Mycoses 2010; 53: 221-226.

10) Shibata N, Okawa Y. Structure of fungal cell wall polysaccharides. Jpn J Med Mycol 2006; 47: 179-184.

11) Watanabe T, Takano M, Murakami M, Tanaka H, Matsuhisa A, Nakao N, Mikami T, Suzuki M, Matsumoto T. Characterization of a haemolytic factor from Candida albicans. Microbiology 1999; 145: 689-694.

12) Calderone RA. Molecular interactions at the interface of Candida albicans and host cells. Arch Med Res 1993; 24: 275279.

13) Rotrosen D, Calderone RA, Edwards JE Jr. Adherence of Candida species to host tissues and plastic surfaces. Rev Infect Dis 1986; 8: 73-85.

14) Iwamoto $L$, Watanabe $T$, Ogasawara A, Mikami T, Matsumoto T. Adherence of Candida albicans is inhibited by yeast mannan. YAKUGAKU ZASSHI 2006; 126: 167-172.

15) Jouault T, Lepage G, Bernigaud A, Trinel PA, Fradin C, Wieruszeski JM, Strecker G, Poulain D. Beta-1,2-linked oligomannosides from Candida albicans act as signals for tumor necrosis factor alpha production. Infect Immun 1995; 63: 2378-2381.

16) Jettanacheawchankit $S$, Sasithanasate $S$, Sangvanich $P$, Banlunara W, Thunyakitpisal P. Acemannan stimulates gingival fibroblast proliferation; expressions of keratinocyte growth factor-1, vascular endothelial growth factor, and type 
I collagen; and wound healing. J Pharmacol Sci 2009; 109: 525-531.

17) Hiragun T, Hide M. Efforts to prevent the onset and exacerbation of atopic dermatitis using food polysaccharides. Journal of Environmental Dermatology and Cutaneous Allergology 2011; 5: 359-364.

18) Han Y, Cutler JE. Antibody response that protects against disseminated candidiasis. Infect Immun 1995; 63: 27142719.

19) Han $Y$, Riesselman MH, Cutler JE. Protection against candidiasis by an immunoglobulin G3 (IgG3) monoclonal antibody specific for the same mannotriose as an IgM protective antibody. Infect Immun 2000; 68: 1649-1654.

20) Villar CC, Kashleva H, Dongari-Bagtzoglou A. Role of Candida albicans polymorphism in interactions with oral epithelial cells. Oral Microbiol Immunol 2004; 19: 262-269.

21) Fujita S. Studies on microtiter broth dilution method for antifungal susceptibility testing of yeast isolates from blood and cerebrospinal fluid. Rinsho Byori 1996; 44: 373-378.

22) Ozaki M, Ohshima T, Mukumoto M, Konishi H, Hirashita A, Maeda N, Nakamura Y. A study for biofilm removing and antimicrobial effects by microbubbled tap water and other functional water, electrolyzed hypochlorite water and ozonated water. Dent Mater J 2012; 31: 662-668.

23) Saijo S, Ikeda S, Yamabe K, Kakuta S, Ishigame H, Akitsu A, Fujikado N, Kusaka T, Kubo S, Chung SH, Komatsu R, Miura N, Adachi Y, Ohno N, Shibuya K, Yamamoto N,
Kawakami K, Yamasaki S, Saito T, Akira S, Iwakura Y. Dectin-2 recognition of alpha-mannans and induction of Th17 cell differentiation is essential for host defense against Candida albicans. Immunity 2010; 32: 681-691.

24) Saijo S, Iwakura Y. Dectin-1 and Dectin-2 in innate immunity against fungi. Int Immunol 2011; 23: 467-472.

25) Shibata N, Kobayashi H, Suzuki S. Immunochemistry of pathogenic yeast, Candida species, focusing on mannan. Proc Jpn Acad Ser B Phys Biol Sci 2012; 88: 250-265.

26) Cambi A, Gijzen K, de Vries JM, Torensma R, Joosten B, Adema GJ, Netea MG, Kullberg BJ, Romani L, Figdor CG. The C-type lectin DC-SIGN (CD209) is an antigen-uptake receptor for Candida albicans on dendritic cells. Eur J Immunol 2003; 33: 532-538.

27) Tamura N, Yoshida T, Miyaji K, Sugita-Konishi Y, Hattori M. Inhibition of infectious diseases by components from Aloe vera. Biosci Biotechnol Biochem 2009; 73: 950-953.

28) Onishi N, Kawamoto S, Ueda K, Yamanaka Y, Katayama A, Suzuki H, Aki T, Hashimoto K, Hide M, Ono K. Dietary pulverized konjac glucomannan prevents the development of allergic rhinitis-like symptoms and $\operatorname{IgE}$ response in mice. Biosci Biotechnol Biochem 2007; 71: 2551-2556.

29) Kawai T, Kase T, Suzuki Y, Eda S, Sakamoto T, Ohtani K, Wakamiya N. Anti-influenza A virus activities of mannanbinding lectins and bovine conglutinin. J Vet Med Sci 2007; 69: 221-224. 